\title{
DIE TRANSCRIPTION DES TAGALOG
}

voN

Dr. JOSÉ RIZAL. *

Als ihr, meine Landsleute, die Dorfschule besuchtet, um das schreiben zu erlernen, oder wenn ihr diese Kunst noch kleineren Bübchen, als ihr es waret, bei zubringen hattet, dann habet ihr ohne Zweifel, so wie es auch mir geschehen, bemerkt, welche Schwierigkeiten es den Kindern bereitete, wenn sie zu den Silben $c a, c e, c i, g a, g u a, g u e, g u i{ }^{\prime}$ u. s. w. kamen, weil sie den Grund zu diesen Unregelmässigkeiten nicht einsahen und nicht sich zu erklären vermochten, warum gewisse Consonanten bald so, bald so ausgesprochen werden sollten. Schläge hagelten nieder, es regnete Strafen, die Händchen bedeckten sich mit Striemen, die ersten Fibelblätter giengen in Fetzen, die Kinder weinten und selbst die Vorzugsschüler mussten mitunter büssen und trotz alle dem kam man aus diesen schrecklichen Thermopylen nicht heraus. Schon damals dachte ich bei mir, dass diese Silben, die so viel Thränen den Knaben entlockten, ihnen ganz unnütz wären, denn in unserer Sprache und in unserer alten Rechtschreibung besitzen wir weder $c e$, noch $c i$, noch ge und $g i^{2}$, weil diese Silben nur dem Spanischen eigenthümlich sind, einer Sprache also, welche von 1000 Knaben nur drei erlernen, wenn sie nach Manila gehn. Doch so sehr ich mich auch fragte, warum man also so etwas lehre und lerne, nach dem doch schliesslich alle nur sich im Tagalischen (einer Sprache, deren Recht-

* Dr. Don José Rizal, selbst ein Tagale, veröffentlichte diesen Artikel in der auschliesslich philippinischen Interessen dienenden Madrider Revue La Solidaridad, Bd. II. Nr. 29, S. 88 u. ff. Diese Abhandlung dürfte nicht nur für Philippinisten, sondern auch für alle Malayisten von Interesse sein. Besagter Artikel trägt als Nebentitel : «Brief an meine Landsleute.» Anmerkung des Uebersetzers. (F. Blumentritt).

1 Spanische Aussprache. Anm. d. Uebersetzers.

2 In Spanischer Aussprache. Anm. d. Uebersetzers. 
schreibung bis jert halb spanisch, halb "ich weiss nicht wie" war) sich ausbilden wollten, so schwieg ich dennoch, weil ich bereits ahnte, dass in meinem Vaterlande Reformen anzuregen so viel heisse, als sich eine böse Suppe einbrocken.

Später, als ich anfieng in meiner Muttersprache Briefe zu schreiben, fielen mir die Widersprüche und Ungereimtheiten der bisher üblichen Orthographie des Tagalog noch mehr in die Augen, aber ich brach dennoch nicht mein Stillschweigen, aus den oben angeführten Gründen.

Als ich aber in eine andere Atmosphäre getreten war und das Werk des Dr. Pardo de Tavera Contribución para el estudio de los antiguos alfabetos filipinos gelesen hatte und damit die Erinnerungen an meine Schulzeit wieder auftauchten, da kam mir doch der Gedanke etwas zu unternehmen, um der Schuljugend Zunächst bei ihren ersten Iuernversuchen ganz unnütze Schwierigkeiten aus dem Wege zu räumen, durch eine Vereinfachung der Rechtschreibung, indem man selbe zugleich den Forderungen des Verstandes und der Logik entsprechend umgestalte, um so die tagalische Orthographie mit dem Geiste dieser Sprache und jenem der Schwesteridiome in besseren Finklang zu bringen und um die Wurzeln besser erkennbar zu machen, auf dass das Studium des Tagalischen nicht allein den Tagalen, sondern auch den Stammfremden erleichtert würde. Ich nahm dem Muth zu diesem Unternehmen einestheils aus den Beobachtungen, die ich in den Volksschulen Sachsens angestellt hatte, wo ich es mit angesehen, welche Mühe die Schullehrer anwandten, um der Jugend den Unterricht zu erleichtern und diesen selbst zu vereinfachen, - andernseits aus dem Umstande, dass es verhältnismässig so wenige tagalische Werke gibt, also noch Zeit zu Reformen ist.

Ich machte mich gleich an meine Aufgabe, und schrieb in der von mir entworfenen Orthographie im September 1886 eine Ubersetzung des Schiller'schen Wilheln T'ell, die sich im Manuscript noch in Kalamba ${ }^{3}$ zugleich mit anderen Uebersetzungen (Märchen von Andersen) befinden dürfte. In einem Romane, den ich im J. 1887 veröffentlichte, liess ich zucrst die tagalischen Worte und Citate, die in demselben vorkommen, in dieser neuen Rechtschreibung drucken, welche über mein Ersuchen bereits einige Freunde anwandten und ich hoffte, dass das philippinische Publikum, nach einer

3 Kalamba ist der Geburtsort Dr. Rizals, es ist ein Städtchen in der Prov. Laguna de Bay (Insel Luzón). 
sachlichen Discussion, die Vortheile und das zeitgemässe der Neuerung anerkennen würde.

Ich war des Glaubens, dass ich der erste Reformator auf diesem Gebiete wäre und hieng noch weiter an diesem Glauben, als ich eine Notiz. des Dr. Pardo de Tavera zu gesichte bekam, welche in den Zeitungen Manilas im J. 1888 veröffentlicht wurde u. die Vortheile der Neuerung begründete und bestätigte. Gleichwohl lebte ich in einem Irrthum.

Monate vor dem Erscheinen meines oben erwälnten Romanes, hatte Dr. Pardo de Tavera bereits sein werk "lil Sanscrito en la lengua Tagaleg" veröffentlicht und in demselben eine Orthographie angewandt, welche die von mir aufgestellte an Vollkommenheit bedeutend übẹrtraf, wenn auch er die seinige bei den citierten Vocabeln nur in Klammern neben die bisher übliche T'ranscription stellte. Das that mir einestheils leid, anderntheils erfüllte es nich mit Freude. Es that mir leid, weil, wenn ich rechtzeitig jenes Werk kennen gelernt hätte (es kam mir erst 1889 unter die Hände), ich das $w$ gerne angewendet haben würde, jenes $w$, welches der genannte Philippinist gebrauchte und dessen ich mich nicht frúher bediente als bis mir der Tagalist Don Pedro Serrano ein gedrucktes Billet eingeschickt hatte, welches die Regein der neuen Schreibweise aufwies. Ich frente mich aber zugleich, weil ich dadurch ersah, dass ich nicht der einzige Erfinder der Idee war, welche beinahe gleichzeitig in unseren Köpfen entstanden war (wemn auch vielleicht das Manuscript des "Sanscrito en la lengua 'Tagalog" vor meiner Übersetzung des Wilhelm Tell niedergeschrieben sein dürfte) und weil das wissenschaftliche Ansehn des Dr. Pardo de Tavera meinen Bestrebungen erheblich zu statten kam. Der beste Beweis, dass beide Reformversuche unabhänig von ein ander und beinahe gleichzeitig unseren Köpfen entsprangen, ohne dass zwischen uns auseinandersetzungen und Besprechungen stattgefunden hätten, liegt eben in dem Gebrauche des $w$, das von Dr. Pardo de Tavera eingeführt worden, von mir aber in meinem oben angeführten Werke noch nicht angewendet worden was, das ich aber sofort nach der Kenntnisnahme adoptierte, weil ich seine ausgezeichnete Nützlichkeit allsogleich einsah.

Ich setze dies deshalb so ausführlich auseiander, damit, wenn min mal die Geschichte dieser tagalischen Orthographie, welche bereits von den gelehrten Tagalisten gebraucht wird, schreibt, man dem Kaiser gebe, was des Kaisers ist, d. h. den tagalischen Studien des Dr. Pardo de Tavera allein dankt man diese Neuerungen, ich bin nur einer ihrer begeistertesten Sendboten gewesen. 
Nun wollen wir aber uns mit den Regeln der neuen Rechtschreibung oder Transcription befassen und werden dann finden, dass es keine fasslichere und einfachere geben kann.

Das alte tagalische Alfabet bestand aus drei Vocalen: $a, i, u$ und vierzehn Consonanten: $k a, g a, \tilde{n g} a, t a, d a, n a, p a$ ba, ma, ya, $1 a, w a, s a$ und ha. Mit diesen 17 Buchstaben konnte man damals, wie heute, alle Worte unserer reichen Sprache niederschreiben.

Der Vocal $a$, welcher am häufigsten vorkommt, hat denselben Klang, wie das castilianische $a$ und so ist über denselben nichts weiter zu sagen.

Das $i$ klingt in der Mitte eines Wortes, wie ein reines spanisches $i$, in einer Schlusssilbe aber entspricht es aber dem englischen $y$ in carry, fluently, etc., d. h. es ist ein Laut, der zwischen $e$ und $i$ liegt. Diese Erscheinung hat viele Spanier glauben lassen, dass die Tagalen $e$ und $i$ nicht zu unterscheiden verstehn, eine Behauptung, die ganz verkehrt ist, da kein Tagale selid für silid setzen würde, ebenso wenig aber siled mit einem so reinen $e$, wie es die Spanier besitzen.

Das $u$ in der Mitte eines Wortes entspricht vollkommen dem spanischen $u$. In der Schlusssilbe nimmt es einen zwischen $o$ und $u$ stehenden Tau an, was ebenfalls einige glauben liess, die Philippiner gebrauchen $o$ und $u$ durch einander, welche Annahme jene köstliche Schreibweise der mönchischen 4 Tagalisten hervorrief, wie sie uns als olo, vondoc u. s. w. entgegentreten. Die Existenz von Worten wie tutoo, noo, poon u. s. w., bei welchen sowohl das erste, wie das zweite $o$ einen zwischen $o$ und $u$ stehenden Laut besitzen, dürfen nicht als eine Ausnahme angesehen werden, denn sie nehmen hier die Stelle eines gedehnten Final $u$ ein. Man kann dem gemïss das o nur am Schlusse eines Wortes zulassen und zwar mit einem Laute $\overparen{\text { ou. }}$.

$T, n, p, b, m, y, l$ und $s$ entsprechen ganz der spanischen Aussprache 5 .

Das $d$ behält seinen Wert, ausgenommen, wenn man es aus euphonischen Gründen mit $r$ vertauscht, wie z. B. in marami, dárating u. s. w. Dieser Ersatz des $d$ durch $r$ ist aber keines wegs der Willkür anheimgestellt, denn das $d$ bleibt $d$ sowohl am Anfange, wie am Schlusse des Wortes und auch in der Mitte, wenn es stummer Consanant ist, so erscheint es denn fehlerhaft tartar, bakor, sinulir fïr

4) Die Mönche sind alle europäische Spanier. Anm. d. Uebersetzers.

5) Das tagalische $y$ entspricht daher dem deutschen $j$. Anm. d. Uebersetzers. 
tatad, bakud, sinulid zu sprechen oder zu schreiben, ebenso wenig wie man roon, rito, raraos für doón, díto, dáraos sagen darf, wie dies in Morong ${ }^{6}$ geschieht.

Dae $h$ ist ein sanfter Hauchlaut, wie bei den meisten Sprachen, es wird im Anfange und in der Mitte des Wortes verwendet, ein Schluss- $h$ gibt es im Tagalischen nicht.

Das gutturale-nasal $\tilde{n g}$ (wie man es bis vor kurzem schrieb), das wir durch Vereinigung beider Buchstaben lieber durch $\tilde{n g}$ wiedergeben würden und welches nach dem Vorschlage des Dr. Pardo de Tavera einfach durch ein $n g$ ersetzt werden sollte, dürfte nach unserem Ermessen leicht und einfach durch $\tilde{g}$ ersetzt werden können und zwar aus folgenden historischen Gründen: In allen Manuscripten und Druckwerken, bis zum XVI. Jahrhunderte, bezeichnete ein Strich oberhalb des Buchstabens ein weggelassenes $m$ oder $n$, wie wir das noch am spanischen $\tilde{n}$ wahrnehmen können, das ja auch ursprünglich nur ein Doppel- $n$ war. Wenn also jenes Strichelchen schon ein $m$ oder $n$ repraesentiert, so würde die alte Schreibweise von ng so viel wie $n n g$ oder $n g n$ bedeuten, was absurd wäre. Wir würden deshalb $n \tilde{g}$ vorschlagen. Aber diese Schreibweise bereitet nicht nur typographische Schwierigkeiten, sie kann vielmehr, wenn der Circumflex nicht über beide Buchstaben gezogen ist, leicht zu Missverstāndnissen führen, deshalb würden wir den Gebrauch des $\tilde{g}$ vorschlagen, wo der gutturale-nasal Laut wiedergegeben werden soll. Hierin bestärkt uns noch die Überzeugung, dass ursprünglich $\tilde{g}$ der Buchstabe füs den gutturale-nasal Laut war, wie wir das sehr gut bei den Abkürzungen der artikel $\overline{n g}$ und $\overline{m g} a$ beobachten können, denn wäre dem nicht so, so würde man es mit $n \overline{n g}$ und $m \overline{n g} a$ wiedergegeben und das abgekürzte $\overline{n g}$ als $n \bar{g} a$ ausgesprochen haben, was, wie alle Tagalen es wissen, unmöglich ist. Man hat später die Combination $\tilde{n g}$ für $\tilde{g}$ gesetzt, als die Mönche, welche den guttural-nasalen Laut selbst nicht aussprechen konnten, ihn bei der Transcription in $n$ und $g$ zerlegten und das Strichelchen ober dem $g$ beibehielten, gleichsam um damit anzudeuten, dass hier die Nasalisation beginne. Der gebrach des $\tilde{g}$ erscheint uns daher sehr empfehlenswert, sowohl aus den angeführten historischen Gründen, als auch der Einfachheit, Bequemlichkeit, Klarheit und Logik willen. So wird, wenn man mangá, bangấ, sangá schreibt, jeder wissen, dan diese Wörte eine Frucht, bez. stossen, einen Stoss parieren heissen und völlig verschieden sind

$\left.{ }^{6}\right)$ Ein District der Insel Luzon. Anm. d. Uebersetzers.

5e Volgr. VIII. 


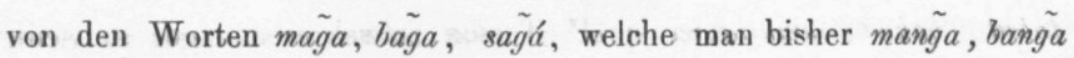
und sanga schrieb und von denen das erste Wort die tagalische Pluralpartikel, das zweite und dritte Krug, bez. Baumast bedeuten. Trotz alledem erscheint uns die Zulassung des $\tilde{n g}$ für den Sohlussnasallaut, der aus der Assimilation der Partikel na (wie in Haring batá für harí na batá) nothwendig, damit der Charakter des assimilierten na nicht verloren gienge. So würden wir am Ende für das von uns schon verworfene $\tilde{n g}$ optieren, obwohl dessen Gebrauch nicht die Vortheile der Einfachheit für sich besitzt, wie das $\tilde{g}$. Hierüber mögen das Endwort die grossen Tagalisten haben.

Bei dem Gebrauche des $g$ oder, besser gesagt, des ga (eines sauften Gutturallautes) haben wir keine andere Neuerung vorzuschlagen, als den Ersatz der alten Silbe gui durch gi. Schon der Umstand, dass wir in unserer Sprache kein aspiriertes $g>$ besitzen, lässt uns die Schreibweise gui als völlig überflüssig erscheinen, sie bringt aber sogar bei der Conjugation jener Zeitwörter, deren Wurzel mit $g$ beginnt, Verwirrung hervor. Z. B.: wir wissen, dass die Mitvergangenheit dadurch gebildet wird, dass man vor den ersten Vocal der Wurzel die Silbe in einschiebt, so wird aus patay: pinatay, aus sipá: sinipa, aus ulan: inulan, aus alís: inalis u. s. w. Nun gut, bei der alten Orthographie müssen wir bei den Verben, welche mit $g a$ oder $g u$ beginnen, eine Änderung der allgemeinen Regel vornehmen, indem wir gezwungen sind zu sagen, dass statt eines einfachen in wir uin vox den ersten Voeal der Wurzel setzen müssen, was ganz absurd und abnormal erscheint, wie in gapas, woraus guinapas gebildet wird, also g-uin-apas. Ganz einfach würde sich die Sache gestalten, wenn man einfach gi für gri setzte, es würde auch hiedurch das erste Lesen den Kindern sehr erleichtern ${ }^{\mathbf{7}}$, die silben $g a, g i, g_{\theta}$ und $g u$ würden doch ihnen leichter zu erlernen seien, als $g a$, GUI, go und $g u$.

Nun wollen wir aber uns mit den Buchstaben $K$ und $W$ beschäftigen, deren Einführung anf so viel Opposition gestossen und zwar nicht allein bei den Ignoranten, sondern auch bei sehr guten Tagalisten. Wir verstehen es einfach nicht, wie so hoch begabte und der Sprache

7) Das spanische $g$ wird bekanntlich vor $e$ u. $i$ wie ch (im Deutschen) ausgesprochen. Soll das $g$ vor einem $e$ oder $i$ den natürlich $g$-Laut behalten, so muss z. wischen $g$ und dem darauf folgenden $\boldsymbol{e}$ oder $\boldsymbol{i}$ ein (stummes) $\boldsymbol{u}$ eingeschoben werden. Die spanischen Mönche haben nun in das Tagalische diese spanische Schreibweise um ihrer Landsleute willen eingeführt $u$. schreiben also guinapas, damit die Spanier es nicht ehinapas aussprächen. Anm. d. Uebersetzers. 
so kundige mäuner, wie der P. Tecson, etwas anstössiges in einer Reform finden konuten, desen einziges Ziel darin bestand dem Tagaliscben sein Eigengepräge zu verleihen, das schreiben und lesen in dieser Sprache möglichst zu vereinfachen, damit auch durch eine klare, wissenschaftliche und logische Orthographie der Wortschatz von Grammatikern -und Philologen leichter bearbeitet werden könnte.

Diejenigen, welche gegen die Einführung des $k$ siç sträuben, begründen ihre Opposition mit deın Hinweise auf die "deutsche Herkunft" dieses Mitlautes. Nun ist es wohl wahr, dass die Deutschen in neuerer Zeit diesem Buchstaben einen besonderen Cultus widmen, indem sie ihn in Worte eingeführt liaben, wohin er nicht hingehört z. B. Konzert, Kursaal u. s. w., aber es heisst sich vor der gesammten Welt grossartig zu blamieren, wenn man dem armen $k$ einen deutschen Ursprung aufınutzen will; wenigstens sollte jeder Mittelschüler wissen, dass das $k$ unverändert aus dem griechischen Alphabet herübergenommen wurde. wo es den nicht ganz unbekannten Namen kappa fülırt. Russen, Engländer, Dänen, Norweger, Schweden, Holländer, ja sogar die Franzosen besitzen diesen "deutschen " Buchstaben und auch die übrigen Völker, welche eine von den turopäischen Alphabeten verschiedene schrift besitzen, haben unter ihren Buchstaben einen, der dem $k$ der lateinischen Transcription entspricht. Es ist demnach, um nicht noch ärgeres zu sageu, kindisch, die Einführung des $k$ in die Transcription des Tagalog unter Hinweis auf die ndentsehe Herkunft" dieses Buchstabens zurückzuweisen und die dbneigung gegen dieses arme $k$ als einen Beweis echten Patriotismus zu nehmen, als ob dieser aueh in den Buchstaben stäke ${ }^{8}$.

Wir wollen nun die Gründe erwigen, welche die Neuerer dazu verleiteten, das $k$ dem alten $c$ u. $q$ vorzuziehn. Zunächst sei, um die Erzpatrioten zu beruhigen, bemerkt, dass das $k$. auch gut spanisch ist, deun es figuriert im spanischen Alphabet zwischen $j$ und $l$ und es ist nicht die schuld des armen $k$, wenn man es nicht gebraucht: wie viele echt-spanische Sachen gibt es nicht in Spanien, wie viele Gesetze, Vorschriften, Verorkinungen, welche noch weniger in Anwendung gebracht werden, als das $k$ ! Aber zum mindesten gebraucht man es in den Worten kiosko, khan, knout, kepi u. s. w.

8 lch lasse hier einige Zeilen weg, in welchen der Autor den Deutschenhass der Spanier, der sich auch gegen den am Carolinenconflict ganz unschuldigen Buchstaben $\boldsymbol{k}$ richtet, in geistreicher Weise verspottet. 
Aber lassen wir den leichten Ton und sagen wir lieber: Das $k$ hat einen festeren Wert als $c$ und $q$ und seine Einführung erspart nicht nur dem Kinde das mühe volle Einlernen der unregelmässigen, verschiedenartigen Aussprache der Silben $c a, c e, c i, c o, c u$ und qua, que, qui, quo, quu, sondern erleichtert auch die grammatische Formelung jener Verba, deren Wurzel mit der Silbe $k a$ oder $k u$ beginnt, wo dann dasselbe gilt, was wir vom gi gesprochen haben. In der That, um das Praeteritum von katay $z$ B. zubilden, so brauchen wir nur die einfache Regel anzuwenden und wir erhalten $K$-ina-tay, während wir früher $c$ in $q$ verwandeln $\mathrm{u}$. das Infix in zu uin umgestalten mussten. ${ }^{9}$

Und, was die Hauptsache ist: die tagalischen Silben $k a, k i, k o$, $k u$ entsprechen gar nicht den spanischen Lauten $c a, q u i, c o, c u$, denn das tagalische $k$ ist leicht aspiriert, also etwa wie $k h$, so zwar dass diejenigen, welche das $k$ nicht aspirieren (es sind dies hauptsächlich die Bewohner Manilas und die Spanier), von den Tagalen Garil 10 genannt werden. Wenn wir nicht jederzeit uns für das einfache und klare einsetzten, so würden wir die Einführung des $k h$ vorschlagen, da aber in unserer Sprache das $k$ immer aspiriert ist, so ist es das allerbeste, das einfache $k$ beizubehalten.

Es bleibt uns noch übrig die Einführung des $w$ zu besprechen, welchem auch so viele einen lebhaften Widerstand entgegengesetzt haben. Wenn man das $y$ annimmt, als ein consonantisches $i$, welches zwei $i$ entsprechen soll wie z. B. in den worten yaman, hahay, biyaya, so muss man consequenter Weise auch das consonantische $w$ annehmen, das zwei $u$ gleichwertig ist, wie in den worten walà, ${ }^{11}$ araw, ${ }^{12}$ kavalá,$^{13}$ ava,$^{14}$ u. s. w., wo das $u$ kein Vocal sondern ein Consonant ist u. auch keinen Diphthong, sondern eine deutliche und perfecte Silbe bildet. Die alte Schreibweise alio anstatt aliw zu schreiben, hat viele der oben erwähnten "garil" verleitet dieses Wort als ali-o auszusprechen, was vollkommen lächerlich ist.

Zum Glück können die Gegner des $w$ nicht als Gegengrund das bedrohte Vaterland anrufen, denn das $w$ hat im Deutschen eine

\footnotetext{
- Ich lasse hier wieder einige, unwesentliche Zeilen weg. Anm. des Uebersetzers.

$10 \mathrm{Im}$ Tagalischen: stotterig, taubstumm, mit sprachfehlern behaftet. Anm. des Uebersetzers.

11 Früher: valì später ualà geschrieben. Anm. d. Uebersetzers.

12 Früher: arau oder arao geschrieben. Anm. d. Uebersetzers.

18 Früher cauald geschrieben. Anm. des Uebersetzers.

14 Früher aua gestrieben. Anm. des Uebersetzers.
} 
dem französischen $v$ entsprechende Aussprache, während unsere [tagalische] jener des englischen $w$ sich nähert. 15

Es gibt noch einen anderen gewichtigen Grund, der für die Einführung des $w$ spricht: es soll das $u$ und $o$ in den alten Transcriptionen von auà und arao z. B. ersetzen, wo der völlige Mangel au Logik der bisherigen Schreibweise hell zu Tage tritt, deun, warum wenden wir bei aù̀ das $u$ und bei arao das $o$, also zwei verschiedene Buchstaben an, um ein und denselben Laut auszudrücken? So etwas muss, wenn nicht Confusion, so doch eine gewisse Unsicherheit hervorrufen, welche demjenigen, welcher lesen lernt, viele Schwierigkeiten verursacht.

Uberdies reimen, in der tagalischen Metrik, die Endsilben ay, $o y$, aw und $i w$ immer consonantisch, nie mit dem Vocalreim, woraus geschlossen werden muss, dass das Gehör des Tagalen nicht des i und $o$, wie man sie bisher an dieser Stelle geschrieben, sondern der consonantischen Lautzeichen $y$ u. $w$ bedarf.

Was die Accentuierung anbelangt, so ist da wenig zu sagen. Die diesbezüglichen, bisher üblichen Regeln sind nicht schlecht, nur über die Accentbezeichnung des langen oder tontragenden Vorals, der sich am Ende vieler Worte, wie in habà, lubhâ etc., vorfindet, wollen wir einige Worte fallen lassen.

Diesen Vocal möge man nach unserem Ermessen mit dem gravis(`) in jenen Worten bezeichnen, bei welchen der lange Schlussvocal nicht den silben- oder tonischen Accent besitzt, wie z. B. in den Worten punò, tamà, pilı̀, wenn aber der tonische Accent mit dem langen Schlussvocal zusammenfällt, wie in den Worten masamá, basâ, masidhó, likó etc. dort möge man das Zeichen des französisehen accent circonflexe ( ${ }^{\wedge}$ ) anwenden, das die Vereinigung Zweier Accente auch figürlich zum Ausdrucke bringt. Diese Schreibweise würde dem schreibenden eine grosse Erleichterung mit sich bringen und wir würden uns nicht mehr genöthigt sehen, zwei accente zu setzen wie dies bisher bei jenen (zweisilbigem) Worten, welche einen langen Schlussvocal besizen, auf den aber der tonische Accent nicht fiel, der Fall war. Anders aber gestaltet sich aber die Sache, wenn es sich um Worte handelt, bei denen der (tonische) Accent auf der dritt vorletzten Silbe ruht, wo wir uns zu der Annahme von zwei Accenten genöthigt

15 Ich lasse hier einige Sätze aus, in welchen der Autor einige von haarsträubender Unwissenheit zeugende Einwürfe der Gegner des $w$ (es sind dies nur spanische Mönche) widerlegt, weil sie für deutsche und holländische Leser ganz überilüssig sind.

Anm. des Uebersetzers. 
sehn, wie z. B. in sásamà, hihigà ect., obwohl hierbeinahe der erste Accent genügte, da diese Worte nicht mit anderen von identischer Form, aber verschiedener Quantität des Schlussvocals, verwechselt werden können, da solche nach unserem Wissen in unserer Sprache nicht existieren.

Bis heute war man gewohnt die Contractionen masdan, palabsin, etc. der Formen masidan, palabasin etc in folgender Weise zu schreiben: mas-dan, palab-sin etc.; wir halten diese Schreibart ungerechtfertigt, denn das Trennungszeichen erweckt in uns den Glauben, dass wir zusammengesetzte Worte, composita, vor uns hätten, was doch unrichtig ist. Nach unserem Ermessen sollte man nach dem Vorgange anderer Sprachen den ausgeworfenen Buchstaben durch einen Apostroph andeuten und so schlagen wir die Schreibweise mas'dán, palab'sín, ting'nun etc. oder die Weglassung sowohl des Trennungszeichens als auch des Apostrophs vor.

Dasselbe schlagen wir für die Substantiv $=$ Partikel $a y$ vor, welche wenn sie an ein mit einem Vocal auslautındes Wort gefügt wird, man immer in: i, umzuwandeln pflegte z. B. aco, $i$, für ako ay. Wir sehen nicht ein, wozu man da zwei Beistriche setzt und das $i$ an statt des $y$ einstellt. Wir würden da einfach einen Apostroph setzen, um das ausgefallene $a$ zu markieren, also in diesem Valle: $a k \hat{y} y$.

Wir zweifeln nicht, dass diese neue Rechtschreibung oder Transcription sich recht bald einbürgern wird und sind überzeugt davon, dass man, bewogen durch ihre Vortheile sie als die nationale, dabei vernunftgemässe und leicht zu erlernende Rechtschreibung unserer harmonischen Muttersprache anerkennen werde. 